\title{
A INCORPORAÇÃO DE RECURSOS DE SIMULAÇÃO NO CURSO DE GRADUAÇÃO EM MEDICINA DA FACULDADE DE MEDICINA DE RIBEIRÃO PRETO - USP
}

\author{
IMPLEMENTING SIMULATION-BASED EDUCATION IN THE MEDICAL UNDERGRADUATE PROGRAM \\ AT THE RIBEIRÃO PRETO FACULTY OF MEDICINE, UNIVERSITY OF SÃO PAULO, BRAZIL
}

\author{
Luiz Ernesto de Almeida Troncon'1 , Cláudia Maria Leite Maffei²
}

\begin{abstract}
'Docente. Departamento de Clínica Médica. Presidente da Comissão de Graduação. ${ }^{2}$ Docente. Departamento de Biologia Celular e Molecular e Bioagentes Patogênicos. Membro Titular da Comissão de Graduação. Coordenadora da Comissão Coordenadora dos Cursos de Medicina e Ciências Biológicas Modalidade Médica. Faculdade de Medicina de Ribeirão Preto - USP.

CORRESPONDÊnCIA: Prof. Dr. Luiz E. A. Troncon. Departamento de Clínica Médica. Hospital das Clinicas da Faculdade de Medicina de Ribeirão Preto - USP. 14048 - 900 Ribeirão Preto, SP - Telefone: (16) 36022457 - Fax: (16 3633 6695). E-mail: ledatron@fmrp.usp.br
\end{abstract}

Troncon LEA, Maffei CML. A incorporação de recursos de simulação no curso de graduação em Medicina da Faculdade de Medicina de Ribeirão Preto - USP. Medicina (Ribeirão Preto) 2007;40 (2): 153-61.

Resumo: A Faculdade de Medicina de Ribeirão Preto (FMRP), desde o início do seu funcionamento em 1952, caracteriza-se pela inovação e pela busca da excelência no desempenho das suas atividades de ensino, pesquisa científica e de assistência à saúde da população. No curso de graduação em Medicina, esta busca tem se refletido em sucessivas mudanças da estrutura curricular e em esforços repetidos para a modernização dos métodos de ensino. Neste sentido, há várias décadas vem sendo introduzidas técnicas de simulação, envolvendo modelos, manequins e simuladores e, em menor escala, o uso de pacientes padronizados. No momento atual, aprovou-se revisão do projeto pedagógico do curso, com redefinição das competências gerais e específicas a serem adquiridas pelos formandos e o estabelecimento de metas a serem atingidas para a renovação dos métodos de ensino, de modo a que sejam oferecidas ao estudante oportunidades de aprendizado mais ativo. Dentre estas metas, prevê-se o investimento em laboratório de habilidades e na difusão das práticas de simulação aplicadas ao ensino e treinamento do estudante.

Descritores: Simulação. Simulação de Pacientes. Competência Clínica. Educação Médica.

\section{1- INTRODUÇÃO}

O ensino superior nas profissões da área da saúde e, em especial, o ensino da Medicina, pela sua própria natureza, caracterizam-se por alto grau de dinamicidade. As mudanças econômicas e sociais que afetam as pessoas cujos problemas de saúde são eventualmente trazidos àqueles profissionais acabam por se refletir nos conteúdos e métodos utilizados na sua educação. Por outro lado, a Educação Médica, sobre- tudo nas últimas quatro décadas, passou a se configurar como uma área multidisciplinar do conhecimento humano em intenso crescimento ${ }^{1 / 6}$. Em razão do grande número de estudos e investigações realizados em seu âmbito, inúmeras transformações e inovações, muitas delas fundamentadas em sólidas evidências ${ }^{2 / 6}$, passaram a ser incorporadas nos currículos dos cursos de Medicina. Dentre elas, encontra-se o emprego de simulações clínicas, que apresentam inúmeras possibilidades de aperfeiçoamento da formação profissional ${ }^{7,8}$. 
A Faculdade de Medicina de Ribeirão Preto (FMRP) da Universidade de São Paulo, desde a criação do curso de Medicina, no início dos anos 1950, tem se preocupado com a qualidade da formação dos seus estudantes. Isto tem se evidenciado por reformulação periódica do seu currículo, bem como por modificações conceituais e metodológicas feitas continuamente pelos professores, responsáveis por disciplinas e departamentos.

Neste artigo, procuramos retomar brevemente alguns aspectos da trajetória da instituição no campo do ensino médico, bem como situar o seu momento atual, em relação às perspectivas de investimento na introdução e na utilização mais intensiva de recursos de simulação clínica no curso de graduação em Medicina da FMRP.

\section{2- CARACTERIZAÇÃO DO ENSINO MÉDI- CO NA FMRP: MODERNIDADE E INO- VAÇÃO}

Desde a sua criação, no final da década de 1940, e o seu início efetivo de funcionamento, em 1952, o curso de Medicina da FMRP associa-se aos conceitos de modernização e inovação. A instituição, que, em seus primórdios constituía-se tão somente do seu curso médico, foi criada em um contexto de um projeto nacional que buscava levar ao interior do país um perfil de modernidade. Assim, configurou-se naquela época como a primeira Faculdade de Medicina a funcionar fora das capitais estaduais ${ }^{9}$.

A concepção da estrutura organizacional da faculdade e do próprio funcionamento do curso médico representou uma das primeiras, se não a primeira, aplicação efetiva no país das proposições do "Relatório Flexner", elaborado nos Estados Unidos no início do século e que transformou radicalmente o ensino médico norte americano ${ }^{5}$. Esse modelo de ensino, em síntese, apresenta as seguintes características: divisão do currículo em básico e clínico, implantação do binômio ensino-pesquisa, ensino clínico desenvolvido predominantemente em hospital universitário e dedicação integral à docência e a pesquisa como regime de trabalho para o corpo docente.

Apesar de, no Brasil, o modelo flexneriano ter passado a receber críticas como modelo de ensino excessivamente centrado em departamentos e exclusivamente desenvolvido no hospital universitário ${ }^{10}$, sua aplicação em instituições sérias, como a FMRP, representou importante evolução conceitual, em que se destacam ao menos dois aspectos que, na década de
1950, podem ser considerados como profundamente inovadores: a profissionalização do corpo docente, caracterizada pela contratação de todos os professores, inclusive os dos departamentos clínicos, em regime de tempo integral, e a institucionalização da investigação científica, como parte do exercício da função docente, ao lado das atividades de ensino médico e de assistência à saúde da comunidade.

Não obstante a adoção do chamado "modelo flexneriano", a FMRP foi também pioneira na implantação do ensino médico fora do hospital, com ações de ensino e assistência médica na comunidade. De fato, o decreto de criação da Faculdade já contemplava a constituição de um "centro de saúde-escola" e, na prática, logo se iniciaram ações específicas de cunho científico, médico e social no então denominado "Instituto de Medicina Rural" no pequeno município de Cássia dos Coqueiros, SP, graças à atuação do Departamento de Medicina Social ${ }^{11}$.

Outro aspecto pioneiro do curso médico na FMRP foi o de contar, desde o seu primeiro desenho no início da década de 1950, com disciplinas e atividades eletivas, inclusive no internato ${ }^{12}$, como um possível reconhecimento da diversidade das oportunidades de formação e da autonomia do educando.

Aspecto distintivo do ensino médico na FMRP é a sua constante preocupação com a qualidade. De fato, desde 1957, ano de formatura da sua primeira turma, registram-se iniciativas de mudança curricular destinadas ao aprimoramento no currículo do curso de graduação em Medicina ${ }^{12,13}$. Como parte de uma destas iniciativas, encontra-se o movimento transcorrido na década de 1980, que resultou na elaboração de um elenco de Objetivos Terminais e Intermediários para o curso médico, aprovados pela Congregação da FMRP em 1987. Este elenco de objetivos constituiu-se em um dos primeiros documentos em que se define o perfil de competências do médico a ser formado, precedendo, em alguns anos, o que passou a ser exigido de todos os cursos, a partir da publicação, em 2001, das Diretrizes Nacionais Curriculares para o curso de graduação em Medicina" ${ }^{14}$. Mais recentemente, a recém criada Comissão Coordenadora do Curso de Medicina (CoC-Med), em conjunto com a Comissão de Graduação (CG), propuseram revisão do projeto pedagógico do curso médico, aprovada pela Congregação da FMRP em 2007, em que os citados objetivos foram revistos e transformados em Competências e Habilidades Gerais e Específicas a serem adquiridas pelos graduandos (Quadros 1 e 2). 
QUADRO 1. Competências e habilidades gerais esperadas do médico a ser formado pela FMRP, de acordo com o novo projeto pedagógico aprovado pela Congregação da unidade em maio de 2007.

\section{COMPETENNCIAS E HABILIDADES GERAIS}

- Identificar as necessidades de saúde, considerando suas dimensões biológicas, psicológicas e sociais, tanto no nível individual quanto no coletivo, por meio de análise crítica, norteada pelo rigor científico, técnico e ético.

- Reconhecer a saúde como estado de bem estar físico, psíquico e social e dependente de condições ambientais favoráveis.

- Reconhecer a necessidade de zelar pela própria saúde física e mental.

- Aplicar, de forma correta e sistemática, os procedimentos rotineiros que conduzem ao diagnóstico e interpretar adequadamente os dados obtidos.

- Indicar e executar, corretamente, as medidas terapêuticas não especializadas das doenças de maior prevalência em nosso meio, bem como dos estados que colocam a vida em risco iminente.

- Contribuir para a promoção da saúde, prevenção das doenças e reabilitação dos incapacitados, levando em conta as condições sócio-econômicas e culturais da comunidade.

- Reconhecer a posição que ocupará no sistema de saúde do país e avaliar, com sentido crítico, como esse sistema atua na solução dos problemas de saúde da população brasileira.

- Estar apto a tomar iniciativas voltadas ao gerenciamento e à administração dos recursos humanos, físicos e materiais nas unidades de saúde onde atua.

- Criar um ambiente de confiabilidade, expresso por comportamento íntegro, comunicação clara, prestação de informações acessíveis e adoção de práticas profissionais pautadas nos princípio da ética.

- Estar apto a assumir posição de liderança, embasada em relacionamento adequado com seus colegas médicos e com membros de outras profissões, atuando com responsabilidade, empatia, tendo em vista o bem estar da comunidade.

- Adquirir, continuamente, informações relevantes à prática médica, de modo a garantir a atualização profissional, através da utilização de recursos de aprendizagem e da análise crítica de comunicações científicas.

- Contribuir para o avanço do conhecimento, realizando observações originais sistematizando sua experiência pessoal e relatando-as apropriadamente, à comunidade médica.

QUADRO 2. Algumas das competências e habilidades específicas esperadas do médico a ser formado pela FMRP, de acordo com o novo projeto pedagógico, que poderiam ser adquiridas mediante o emprego de simulações clínicas.

COMPETÊNCIAS E HABILIDADES ESPECÍFICAS

- Relacionar-se adequadamente com o paciente e seus familiares reconhecendo os fatores psicológicos que possam estar envolvidos na doença atual.

- Obter e redigir, de forma clara, uma história clínica que contenha as queixas apresentadas pelo paciente, a evolução de sua doença, seus antecedentes pessoais e familiares, bem como uma descrição de seus hábitos e de suas reações à doença.

- Realizar o exame físico, de acordo com as técnicas semiológicas de inspeção palpação, percussão e ausculta. Saber empregar os instrumentos de uso comum no exame físico.

- Realizar a coleta de material para os exames laboratoriais freqüentemente necessários e de pequena complexidade técnica.

- Indicar e executar, corretamente, as medidas terapêuticas necessárias para manter as funções vitais em caso de urgência médica.

- Realizar procedimentos cirúrgicos de pequena complexidade com fins terapêuticos

- Diagnosticar a gravidez, acompanhar a sua evolução, efetuar um parto normal e fazer o encaminhamento adequado de gravidez de alto risco.

- Avaliar corretamente o desenvolvimento físico e mental do ser humano, tomando as medidas adequadas para prevenção e correção dos desvios da normalidade mais freqüentes e acidentes e propor medidas de prevenção primária, secundária e terciária. 
Outra iniciativa inovadora da FMRP, implantada no âmbito da reestruturação curricular aprovada em 1992, foi a criação de um Programa de Avaliação Terminal do Desempenho dos Graduandos ${ }^{15}$. Mediante a realização de provas específicas, compunha-se um perfil de domínio, pelos graduandos, de habilidades cognitivas, psicomotoras e de atitudes que indicava o grau de consecução dos objetivos educacionais definidos pela instituição, para o curso de graduação em Medicina ${ }^{16,17,18}$.

A reestruturação implantada na FMRP em 1993 apresentava como linhas gerais não só a racionalização do conteúdo e da carga horária ministrados, mas também a integração de conteúdos das várias disciplinas tradicionais em grandes unidades de ensino orientadas por órgãos e sistemas ou por mecanismos de regulação ou etio-patogênicos, entre outras inovações ${ }^{19}$. O desenho curricular era altamente propício para a gradual substituição do ensino didático tradicional para modalidades menos diretivas, que implicasse na utilização de métodos mais ativos e integradores de aprendizagem ${ }^{4}$. No entanto, isto acabou por não acontecer, por dificuldades de interação entre docentes de diferentes áreas, e as subseqüentes propostas de reorganização, levadas a cabo em 2001 e 2007, ainda que corrigindo distorções e aprimorando a ministração do ensino, acabaram por consolidar o modelo tradicional, uma vez que se retornou, em grande parte, ao esquema de disciplinas estanques e se manteve a predominância do aprendizado passivo.

\section{3- ESFORÇOS PARA A MODERNIZAÇÃO DOS MÉTODOS DE ENSINO NO CURSO MÉDICO NA FMRP}

Não obstante as iniciativas inovadoras introduzidas no curso médico da FMRP, a ministração do ensino tem se caracterizado pela predominância de métodos tradicionais e pela dificuldade de difusão de modelos inovadores propostos, muitas vezes, pela iniciativa individual de professores. De fato, em muitas das disciplinas notam-se iniciativas de prover o ensino menos diretivo e mais estimulador da participação ativa do estudante. No entanto, predomina, em ambos os atuais ciclos básico e clínico, o emprego dos métodos tradicionais e, sobretudo, o da aula teórica formal. Existe, portanto, clara tendência de valorização da transmissão do conhecimento sobre a preocupação com o desenvolvimento de habilidades de aprendizado contínuo. Preocupadas com este estado, na recente proposta de revisão do projeto pedagógico do curso, foi incluído um conjunto de metas a serem atingidas, que são resumidas no Quadro 3. Estas metas visam, em grande parte, a renovação dos métodos de ensino e aprendizagem, de modo a que sejam oferecidas ao estudante oportunidades de participação mais ativa na incorporação do conhecimento e no aprendizado das habilidades. A consecução desta meta demanda a capacitação dos professores para o uso de novos recursos e técnicas de aprendizado, incluindo, entre outras, as metodologias "problematizadoras", os jogos educativos e o ensino à distância, com a utilização de meios eletrônicos de comunicação e de acesso às fontes de informação ("e-learning"). Neste sentido, justificase a meta de investimento em um Laboratório de Habilidades e na difusão das práticas de simulação aplicadas ao ensino e ao treinamento do estudante.

Com o uso de técnicas de simulação, o estudante tem a oportunidade de adquirir habilidades variadas, repetindo os procedimentos diagnósticos ou terapêuticos tantas vezes quanto forem necessárias até atingir o estágio necessário de domínio. A execução da tarefa pode e deve ser observada, de modo que as devidas correções sejam feitas de imediato e o estudante receba os devidos comentários ("feedback") sobre a sua atuação. Operando em condições de simulação, o constrangimento ao estudante que se encontra em fase inicial do treinamento é consideravelmente minimizado e, em especial, o processo de aprendizado, envolvendo a prática repetida, não vai representar risco ou desconforto para pacientes reais. Além disso, o emprego das técnicas de simulação permite que se ofereçam as mesmas oportunidades de aprendizado, prática e treinamento para todos os estudantes, de forma mais homogênea, sem depender das circunstâncias e do acaso envolvidos no aprendizado baseado em situações reais. Deste modo, o estudante vai se encontrar muito mais preparado quando, na etapa de treinamento em serviço, se defrontar com situações reais em que vai precisar executar o procedimento diagnóstico e terapêutico.

Estas considerações, que se aplicam inteiramente ao aprendizado e ao treinamento para a execução de procedimentos específicos envolvidos com o diagnóstico e, sobretudo, com o tratamento, envolvendo modelos anatômicos, manequins e simuladores variados ${ }^{20}$, também são, em grande parte, pertinentes à aquisição de habilidades de comunicação e, eventualmente, de exame físico, que envolvem as simulações com pacientes simulados ou padronizados ${ }^{21}$. Destaque-se que a utilização de simulações, em geral, vem sendo considerada como um poderoso fator de redução de erros e de melhora do desempenho profissional ${ }^{8}$. 
QUADRO 3. Metas a serem atingidas no âmbito do novo projeto pedagógico do curso de Medicina da FMRP, aprovado pela Congregação da Faculdade em 2007

\section{METAS DO CURSO DE MEDICINA}

- Renovação dos métodos de ensino, de modo a que sejam oferecidas ao estudante oportunidades de aprendizado mais ativo.

- Capacitação dos professores para o uso de novos recursos e técnicas de aprendizado.

- Introdução de novos métodos (problematização, jogos educativos e ensino à distância, com a utilização de meios eletrônicos de comunicação e acesso às fontes de informação).

- Investimento em laboratório de habilidades e na difusão das práticas de simulação aplicadas ao ensino e treinamento do estudante.

- Revisão contínua dos conteúdos, de modo a evitar excessivo detalhamento e a representar esforço mantido para articular ou integrar conteúdos e práticas.

- Manter os conhecimentos teóricos e práticos essenciais à formação das competências e habilidades gerais que formam o perfil de formação desejado.

- Proporcionar ao aluno condições de aprendizado mais participativo, com elaboração do calendário didático e da grade de distribuição de turmas de modo a que possibilitem a formação de grupos menores de estudantes.

- Organização das atividades do internato de modo a contemplar maior carga de trabalho em unidades de atenção primária e secundária, mais do que no hospital universitário de nível terciário.

- Implementação de mudanças nas práticas de avaliação, de modo a contemplar domínios outros, que não somente o cognitivo, bem como a privilegiar olhares externos a quem ministra as várias disciplinas.

- Implementação de práticas de efetiva educação interdisciplinar e multi-profissional, com a articulação das atividades dos alunos de Medicina com as dos estudantes de outros cursos da unidade e do campus.

\section{4- INTRODUÇÃO DE SIMULAÇÕES NO ENSINO DE GRADUAÇÃO NA FMRP}

A história da introdução de simulações no ensino médico da FMRP é de difícil reconstituição, mas é possível afirmar que, no final dos anos 1970, já eram utilizados modelos anatômicos e manequins no aprendizado de algumas habilidades, como a recepção do recém-nascido e assistência ao parto normal, no âmbito, respectivamente, dos Departamentos de Puericultura e Pediatria e de Ginecologia e Obstetrícia. É, também, certo que no final dos anos 1980 já se utilizavam manequins para o ensino de habilidades ligadas à tubagem traqueal, à assistência ao traumatizado, bem como para o aprendizado de manobras envolvidas na reanimação cardiorespiratória. Nos anos 1990, durante o desenvolvimento do Programa de Avaliação Terminal do Graduando, foram utilizados continuamente manequins para as habilidades de acesso venoso, recepção ao recém-nascido, exame ginecológico, assistência ao parto normal, acesso às vias aéreas e reanimação cardiorespiratória ${ }^{15}$.

A criação das disciplinas de "Iniciação à Saúde" e de "Primeiros Socorros e Atendimento PréHospitalar", dentro do conceito adotado na reestruturação curricular de 1993 de introdução mais precoce do estudante à problemática da saúde e ás práticas mais habituais de assistência ${ }^{19}$ veio possibilitar o emprego mais rotineiro, já no primeiro e no segundo anos do curso de Medicina, de manequins de simulação para o aprendizado de técnicas de acesso venoso, de administração de injeções intramusculares e de manuseio da pessoa acidentada.

Esta década registra, ainda, a introdução na FMRP, em 1995, de técnicas de simulação com "pacientes padronizados", na prova final da disciplina de Semiologia do Departamento de Clínica Médica ${ }^{22}$ e, pouco tempo mais tarde, na prova de Habilidades Clínicas do mencionado Programa de Avaliação Terminal do Graduando ${ }^{15}$. Os "pacientes padronizados" constituem um técnica caracterizada pelo emprego de pessoas normais ou pacientes reais, devidamente preparados e treinados para protagonizar situações clínicas para fins de ensino e de avaliação ${ }^{23}$.

Ainda que não se disponha de registros de resultados da avaliação sistemática do impacto da introdução de recursos de simulação no ensino de graduação da FMRP, é impressão corrente que tanto os estudantes como os professores têm recebido muito po- 
sitivamente estas inovações. Isto é também atestado, ainda que indiretamente, pela persistência e o crescimento destas iniciativas no seio da instituição.

\section{5- CRIAÇÃO DO LABORATÓRIO DE TREI- NAMENTO EM PROCEDIMENTOS MÉ- DICOS GERAIS NA FMRP}

No contexto da renovação dos métodos de ensino e de maior difusão de recursos de simulação no curso de graduação em Medicina da FMRP é que se propõe a criação e o funcionamento de um Laboratório de Treinamento em Procedimentos Médicos Gerais, a ser instalado, ao menos inicialmente, no Laboratório Multidisciplinar da instituição. O ensino do conteúdo programático das disciplinas do ciclo básico dos vários cursos de graduação da FMRP está concentrado em laboratórios e salas de aula do edifício denominado Laboratório Multidisciplinar (LMD), que abriga laboratórios de Anatomia, Microscopia, Histotecnologia, Bioquímica, Fisiologia, Cinesiologia, Fisioterapia e Terapia Ocupacional integradas e aplicadas à infância. Estes laboratórios encontram-se plenamente equipados, dispondo ainda de facilidades de apoio, como salas para preparo de materiais.

O LMD dispõe, também, de anfiteatros grandes e de salas de aulas menores para seminários e atividades em pequenos grupos, todas devidamente equipadas com computadores e sistemas de projeção. Integra o LMD as salas do programa "Pró-aluno", que permite o acesso do estudante a computadores interligados a sala de apoio onde ficam as impressoras, scanner e gravadores de discos.

Em futuro muito próximo, será instalado no LMD um sistema de vídeo-conferência integrado a salas do Hospital das Clinicas, especificamente, seis salas do Centro Cirúrgico, duas salas do ambulatório de Clínica Médica, uma sala do ambulatório de Ginecologia (filmadora no colposcópio), uma sala no ambulatório de Pediatria e uma sala do ambulatório de Psiquiatria. Esse sistema permitirá que vários alunos possam desfrutar de experiências que na prática seriam inviáveis pela ética e segurança (excesso de alunos numa sala de centro cirúrgico ou em ambulatórios). Além disso, proporcionará aos docentes discutir tópicos como: relacionamento médico-paciente, posturas éticas e humanas, técnicas e mesmo revisar temas diversas vezes, pela gravação de imagens. Os arranjos para a articulação de serviços com o Hospital das Clínicas da FMRP e a própria compra dos equi- pamentos para a instalação desse sistema já se encontram em fase adiantada.

É no espaço físico do LMD que se reservou área física onde se pretende implantar o Laboratório para Treinamento de Procedimentos Médicos Gerais. Para este laboratório prevê-se a aquisição de manequins apropriados para o aprendizado de certas habilidades motoras, como aplicação de injeção intramuscular, coleta de sangue de veia do braço, realização de toque ginecológico e exame da próstata. Prevê-se, também, a aquisição de manequins e de modelos para exame urológico e de mamas, cateterismo vesical, cuidados com traqueotomia e colostomia, intubação de vias aéreas e drenagem de tórax e reanimação do paciente com parada cardiorespiratória. Os recursos para tanto já foram solicitados á Pró-Reitoria de Graduação da Universidade de São Paulo, no âmbito de programa específico ("Pró-Lab") de investimento em infra-estrutura de laboratórios didáticos.

Uma vez instalado e plenamente equipado, este laboratório poderá ser utilizado em várias atividades do curso de Medicina, como nas aulas teórico-práticas das disciplinas dos primeiros anos, como Iniciação à Saúde e Primeiros Socorros e Atendimento PréHospitalar. Nos anos mais avançados, o laboratório poderá ser utilizado nas atividades das disciplinas clínicas integradas, como Sistema Cardiovascular, Sistema Respiratório, Sistema Urinário, bem como no ensino de Ginecologia e Obstetrícia, Anestesiologia, Emergências Médicas e Terapia Intensiva. Pode, ainda, propiciar a realização de atividades práticas conjuntas e comuns a diferentes áreas, ensejando a integração de conhecimentos e habilidades relacionados às várias áreas do currículo. A instalação e o pleno equipamento deste laboratório permitirão, ainda, o treinamento de alunos em seus horários livres, sob supervisão de técnicos especializados.

Em etapa futura, poderá ser factível a implantação de um centro local de recrutamento, seleção e treinamento de pacientes simulados para aquisição de habilidades de comunicação, simulação de consultas e treinamento geral em competências que envolvem a interação com pessoas. Estas atividades poderão ser articuladas com os recursos de vídeo-conferência a ser instalados conectando as dependências da FMRP com locais específicos do Hospital das Clínicas da FMRP.

A FMRP orgulha-se em proporcionar aos seus estudantes amplas oportunidades de treinamento prático, com supervisão especializada, em seus diversos 
locais de atenção, como Unidades Básicas de Saúde, Núcleos do Programa de Saúde da Família, Centro de Saúde Escola, Hospital Maternidade "Mater", Unidade de Emergência e no seu Hospital das Clínicas. Porém a divisão dos alunos em grupos menores para melhor aproveitamento e adequação dos serviços, nem sempre permite igualdade de oportunidades a todos.

A idéia desse novo Laboratório para Treinamento de Procedimentos Médicos Gerais é oferecer as mesmas condições para o aprendizado de habilidades e a atitudes a todos os alunos e faz parte do projeto de melhorias no ensino, representando importante inovação para os cursos de graduação. A sua implementação corresponderá, portanto, à consecução de uma das metas da Comissão de Graduação, relativa à renovação dos métodos de ensino e de aprendizado dos vários cursos da unidade, que consta formalmente do novo projeto pedagógico do curso de Medicina. Poderá ser utilizado por todas as disciplinas, nos treinamentos de procedimentos tanto propedêuticos quanto terapêuticos, bem como para aquisição de outras competências envolvidas no relacionamento com os pacientes. Além de humanizar o ensino, os professores poderão verificar com muito mais facilidade as reações dos alunos em diversas situações. Como resultado previsível da utilização deste novo Laboratório, os alunos poderão atingir nível adequado de treinamento, antes de terem as oportunidades de trabalhar com o paciente real.

Cumpre-se, no entanto, ressaltar que os manequins e os vários recursos de simulação não substituem os pacientes, apenas antecedem o contato com eles, de modo seguro, complementando e otimizando $\mathrm{o}$ aprendizado.

\section{6- PERSPECTIVAS}

O desenvolvimento bem sucedido do que se propõe vai depender da resultante de um conjunto de fatores contrários e favoráveis ao investimento de tempo, energia e recursos financeiros no aperfeiçoamento do ensino de graduação. Dentre os fatores contrários, deve ser mencionada a pouca efetividade das políticas nacionais de melhoria do ensino médico atualmente existente. $\mathrm{O}$ estabelecimento das diretrizes nacionais curriculares para o curso de Medicina ${ }^{14}$, especialmente no que se refere aos objetivos e conteúdos, devem ser considerados como importantes avanços, mas, por outro lado, os mecanismos de estímulo às escolas para sua implementação, restringemse a aspectos específicos da interação com o Sistema
Nacional de Saúde ${ }^{24}$. Não há previsão de restrições aos cursos que não as implementarem e a busca de adequação a estas diretrizes sem a devida estruturação por parte das escolas médicas, especialmente as privadas, implica em que o ensino junto aos serviços de saúde, possa vir a ser desenvolvido sem a devida supervisão qualificada. Isto pode vir a comprometer a qualidade da formação dos egressos, ao invés de melhorar o ensino médico.

No âmbito da universidade, embora não haja uma política clara de renovação dos métodos de ensino e aprendizagem, são permanentes os esforços para a valorização do trabalho docente na graduação ${ }^{25}$, o que inclui o investimento em atividades de capacitação e atualização dos professores em conceitos e métodos inovadores ${ }^{26}$. Ainda nesta esfera, é importante ressaltar a existência de programa permanente de manutenção e investimento em infra-estrutura de laboratórios específicos para o ensino de graduação ("Pró-Lab"). É importante, porém, mencionar que a escassez ou ausência de mecanismos institucionais de reconhecimento e de incentivo do trabalho docente na graduação não é uma característica nacional ou local, mas parece prevalecer em todo o mundo ${ }^{27}$.

Por outro lado, a Universidade de São Paulo e a FMRP concedem a seus professores ampla liberdade de tomar iniciativas inovadoras em todas as atividades acadêmicas, embora nem sempre o investimento pessoal de tempo e de energia no ensino seja reconhecido em termos de progressão na carreira, se não vier acompanhado de indicadores mais concretos de produção científica publicada em veículos de impacto. Neste sentido, parece-nos pertinente estudar mecanismos, como a criação de um Centro de Simulações Clínicas, que possa agregar todas as iniciativas isoladas de uso de recursos de simulação para o ensino na FMRP. Esta medida, além de unificar e agregar os esforços poderia racionalizar a administração dos recursos, otimizando a sua utilização. A eventual criação deste Centro poderia, sobretudo, prover condições de desenvolvimento de investigações científicas neste campo do ensino baseado em simulações, afeito à área do conhecimento da Educação Médica. A criação deste Centro nos parece especialmente pertinente se aceitarmos como correta a previsão do crescimento das iniciativas individuais do uso de recursos de simulação, que se insere no movimento crescente e cada vez mais difundido em todo o mundo para o ensino e o treinamento das habilidades clínicas em bases mais racionais e efetivas. 
Troncon LEA, Maffei CML Implementing simulation-based education in the medical undergraduate program at the Ribeirão Preto Faculty of Medicine, University of São Paulo, Brazil. Medicina (Ribeirão Preto) 2007;40 (2): 153-61.

Abstract: The Ribeirão Preto Faculty of Medicine (FMRP), since the early days of its medical undergraduate program in 1952, may be characterized as an innovative institution in the search of excellence in teaching, scientific research and health assistance. The FMRP medical undergraduate program underwent successive changes in its curriculum structure, and has hosted repeated efforts for modernization and improvement in teaching and learning methods. In this context, a number of simulation techniques have been introduced throughout the last decades, which included the utilization of plastic models, mannequins and simulators and, at lesser extent, of simulated, standardized patients for teaching and assessment. A revision of the educational plan for the medical undergraduate program has been recently approved, with a redefinition of the general and specific skills and competences to be achieved by medical graduates. This new plan includes a set of objectives to be pursued in the near future, which includes the continuous improvement in teaching and learning methods aiming at providing conditions for a more active learning. This set of objectives includes the investment for the implementation of a skills laboratory, and for the diffusion of simulation techniques.

Keywords: Simulation. Patient Simulation. Clinical Competence. Education, Medical.

\section{REFERÊNCIAS}

1 - Kassirer JP. Redesigning Graduate Medical Education Location and Content. N Engl J Med 1996; 335:507-9.

2 - Norman G. Research in medical education: three decades of progress. Br Med J 2002; 324: 1560-2.

3 - Wass V, Richards T, Cantillon P. Monitoring the medical education revolution. Br Med J 2003; 327:1362.

4 - Ludmerer KM. Learner-Centered Medical Education. N Engl J Med 2004; 351:1163-4.

5 - Cooke M, Irby DM, Sullivan W, Ludmerer KM. American medical education 100 years after the Flexner report. N Engl J Med 2006; 355:1339-44.

6 - Dauphinee WD, Wood-Dauphinee S. The need for evidence in medical education: the development of best evidence medical education as an opportunity to inform, guide, and sustain medical education research. Acad Med 2004; 79: 92530.

7 - Bradley P. The history of simulation in medical education and possible future directions. Med Educ 2006; 40: 254-62.

8 - Ziv A, Ben-David S, Ziv M. Simulation based medical education: an opportunity to learn from errors. Med Teach 2005; 27:193-9.

9 - Amorim DS. Faculdade de Medicina de Ribeirão Preto: 1952 - 1992 (Editorial). Medicina (Ribeirão Preto) 1992; 25: 1-8.

10 - Gonçalves EL. A medicina no Brasil, hoje: ensino e prática. Rev Hosp Clin Fac Med Univ Säo Paulo 1990; 45: 190 -3.

11 - Yazlle-Rocha JS. Departamento de Medicina Social: A reestruturação e os novos tempos. Medicina (Ribeirão Preto) 2002; 35: 306-12.
12 - Magrini ED, Martinez R. O curso de Medicina e as comissões coordenadoras da graduação na Faculdade de Medicina de Ribeirão Preto: uma retrospectiva. Medicina (Ribeirão Preto) 2002; 35: 385- 8.

13 - Figueiredo JFC, Rodrigues CRC. Estratégias para a reformulaçäo da estrutura curricular na Faculdade de Medicina de Ribeiräo Preto na última década e estágio atual do processo. Rev Bras Educ Med 1990; 14: 20-3.

14 - Brasil, Ministério da Educação. Resolução CNE/CES № 4, de 7 de Novembro de 2001: Diretrizes Nacionais Curriculares para o curso de graduação em Medicina. Disponível em http://portal.mec.gov.br/cne/arquivos/pdf/CES04.pdf.

15 - Troncon LE, Figueiredo JF, Rodrigues MLV, Peres LC, Cianflone AR, Piccinato CE, Colares MFA. Implantação de um programa de avaliação terminal do desempenho dos graduandos para estimar a eficácia do currículo na Faculdade de Medicina de Ribeirão Preto. Rev Assoc Med Bras 1999; 45: 217-24.

16 - Piccinato CE, Figueiredo JF, Troncon LE, Peres LC, Cianflone AR, Colares MFA, Rodrigues MLV. Avaliação de objetivos educacionais em duas diferentes estruturas curriculares Faculdade de Medicina de Ribeirão Preto, Universidade de São Paulo, Brasil. Rev Assoc Med Bras 2004; 50: 68-73.

17 - Figueiredo JF, Troncon LE, Rodrigues MLV, Cianflone AR, Colares MFA, Peres LC, Piccinato CE. Effect of curriculum reform on graduating student performance. Med Teach 2004; 26: 244-9.

18 - Troncon LEA, Figueiredo JFC, Rodrigues MLV, Piccinato CE, Peres LC, Cianflone ARL, Colares MFA. Avaliação de uma reestruturação curricular na Faculdade de Medicina de Ribeirão Preto: influência sobre o desempenho dos graduandos. Rev Bras Educ Med 2004; 28: 145-55. 
19 - Prado WA. Desenvolvimento e implantaçäo da nova estrutura curricular na Faculdade de Medicina de Ribeiräo Preto: dificuldades e avanços. Medicina (Ribeiräo Preto) 1996; 29: 373-82.

20 - Pazin-Filho A. Simulação: definição. Medicina (Ribeiräo Preto) $2007 ; 40(2): 162-6$.

21 - Troncon LEA. Utilização de pacientes simulados no ensino e na avaliação de habilidades clínicas. Medicina (Ribeiräo Preto) 2007; 40 (2 ): 180-91.

22 - Troncon LEA, Foss NT, Voltarelli JC, Dantas RO. Avaliação de habilidades clínicas por exame objetivo estruturado por estações, com emprego de pacientes padronizados: uma aplicação no Brasil (Parte II). Rev Bras Educ Med 1996; 20: 53-60.

23 - Barrows HS. An overview of the uses of standardized patients for teaching and evaluating clinical skills. Acad Med 1993; 68: 443-51.
24 - Brasil. Ministério da Saúde. Programa Nacional de Reorientação da Formação Profissional em Saúde - PróSaúde. Disponível em http://portal.saude.gov.br/saude/ visualizar_texto.cfm?idtxt=22978.

25 - Brasil. Universidade de São Paulo. Diretrizes da Pró-Reitoria de Graduação - Biênio 2002/2003. Disponível em http:// www.usp.br/prg/proreit/gestao2002.htm.

26 - Brasil. Universidade de São Paulo. Pró-Reitoria de Graduação. Portaria Interna 04/2004 (criação do Grupo de Apoio Pedagógico).

27 - Biggs JS, Price DA. Sustaining and rewarding clinical teaching. Med Educ 1992;26:264-8. 